\title{
Expression and role of miR-338-3p in peripheral blood and placenta of patients with pregnancy-induced hypertension
}

\author{
$\mathrm{JUN} \mathrm{LI}^{1}$, YAN WU ${ }^{2}$ and HUI LIU ${ }^{1}$ \\ ${ }^{1}$ Fetal Heart Monitoring Unit, ${ }^{2}$ The Fifth Department of Obstetrics and Gynecology, \\ Laiwu Maternal and Child Health Hospital, Laiwu, Shandong 271100, P.R. China
}

Received September 18, 2018; Accepted November 11, 2019

DOI: $10.3892 /$ etm.2020.8719

\begin{abstract}
The present study aimed to investigate the role of miR-338-3p in pregnancy-induced hypertension (PIH), and its effects on human trophoblast cells in vitro. Quantitative real-time PCR was used to detect miR-338-3p expression. Human trophoblast HTR8/SVneo cells were transfected with miR-338-3p mimics. Effects of miR-338-3p on cell proliferation, invasion and metastasis, and anoikis resistance were detected by CCK-8 assay, Transwell chamber assay, flow cytometry and western blot analysis, respectively. Bioinformatics analysis was performed to predict the target of miR-338-3p, and the results were confirmed by dual luciferase reporter assay. The expression level of miR-338-3p was significantly upregulated in the peripheral blood and placenta of PIH patients. CCK- 8 assay showed that miR-338-3p mimics inhibited the proliferation of HTR8/SVneo cells at indicated time points. Flow cytometry showed that miR-338-3p transfection significantly increased the Ki-67 expression in the HTR8/SVneo cells, indicating enhanced cell proliferation. Transwell chamber assay and western blot analysis showed that the invasion and metastatic abilities of the HTR8/SVneo cells were significantly decreased in the miR-338-3p transfection group, as well as expression levels of MMP-2 and MMP-9. Bioinformatics analysis and dual luciferase reporter assay indicated that $A K T 3$ is a target gene of miR-338-3p. Our results suggest that miR-338-3p is significantly increased in the peripheral blood and placenta of $\mathrm{PIH}$ patients, which is correlated with the disease development. miR-338-3p inhibits proliferation, invasion and metastasis, and apoptosis resistance of human trophoblast cells by targeting AKT3.
\end{abstract}

\section{Introduction}

Pregnancy-induced hypertension (PIH) is an unexplained multi-systemic disease during pregnancy, and is a clinical

Correspondence to: Dr Jun Li, Fetal Heart Monitoring Unit, Laiwu Maternal and Child Health Hospital, 12 Fengcheng West Street, Laiwu, Shandong 271100, P.R. China

E-mail: jljljlj1333@sohu.com

Key words: miR-338-3p, pregnancy-induced hypertension, AKT3, peripheral blood, placenta obstetric issue $(1,2)$. PIH induces a high and uncontrollable incidence of maternal and perinatal morbidity, and ranks as the leading cause of maternal and perinatal deaths worldwide, especially in developing countries $(3,4)$. Although the pathogenesis of PIH is still unclear, it has been widely accepted that the placenta may play an important role in the disease development. Clinical symptoms of PIH are found to gradually disappear after the placenta is excreted. Therefore, termination of pregnancy is currently one of the treatment options for PIH $(5,6)$. In recent years, placental trophoblast cells have been thought to be closely related to the pathogenesis of PIH. Placental trophoblast cells are important for embryonic implantation, placental remodeling and oxygen supply $(7,8)$. Insufficient trophoblast cell invasive ability during pregnancy often leads to impaired development of the placental vascular network, which induces placental ischemia/hypoxia and causes PIH, consequently threatening the safety of the pregnant woman and fetus (9). Therefore, it is of great importance to investigate the molecular mechanisms of trophoblast cell proliferation and infiltration to understand the pathological changes of placenta in PIH, which might contribute to strategies for disease treatment.

MicroRNAs (miRNAs or miRs) are a class of non-coding small RNA molecules consisting of 18-22 nt, which bind to the 3'-untranslated region (UTR) of mRNAs to inhibit the translation process. miRNAs play important roles at the post-transcriptional level (10). Previous studies have shown that miRNA molecules are involved in almost all pathophysiological changes and functions in a variety of diseases, such as hypertension, tumors and diabetes (11). It has been demonstrated that the expression levels of various miRNAs are significantly altered in the development of PIH, including miR-210 and miR-204-5p (12,13). Moreover, it has also been demonstrated that miRNAs are involved in the regulation of the biological behavior of placental trophoblast cells. Wang et al (14) found that miR-362-3p regulates the proliferation and metastasis of trophoblast cells through the hypoxia-induced PAX3 pathway.

At present, the regulatory process of miRNAs in the biological behavior of placental trophoblast cells still needs to be elucidated. miR-338-3p is located in the intron of apoptosis-associated tyrosine kinase 7 , which has been recognized as a tumor-associated miRNA molecule in recent years (15). Moreover, previous studies have shown that miR-338-3p is abnormally expressed in various tumors (such as ovarian 
cancer, malignant melanoma and hepatocellular carcinoma), and participates in the regulation of tumor cell proliferation and metastasis $(16,17)$. During pregnancy, the biological behavior of trophoblast cells has certain similarities with tumor cells, including enhanced invasive ability, and the remodeling of the microenvironment and vascular network (18). In the present study, the effects of miR-338-3p on the development of trophoblast cells and its involvement in PIH pathogenesis were investigated. The expression and function of miR-338-3p in the placental tissues of PIH were quantified and analyzed by quantitative real-time PCR, western blot analysis, Transwell assay and flow cytometry, respectively.

\section{Materials and methods}

Collection of tissue specimens. A total of 38 subjects with PIH who underwent Caesarean section in our hospital, from January 2016 to December 2017 were included in this study. Moreover, another 26 normal delivering females were recruited as control during the same period. For these subjects, placental tissues and peripheral blood samples $(5 \mathrm{ml})$ were collected. For the hypertension disorder complicating pregnancy (HDCP) group, there were 21 cases of PIH (PIH; elevated blood pressure after 20 weeks of gestation, and no proteinuria), 13 cases of mild pre-eclampsia (mPE; systolic blood pressure $\geq 140 \mathrm{mmHg}$, or diastolic blood pressure $\geq 90 \mathrm{mmHg}$, and 24-h proteinuria $\geq 300 \mathrm{mg}$ ), and 4 cases of severe pre-eclampsia (sPE; systolic blood pressure $\geq 160 \mathrm{mmHg}$, or diastolic blood pressure $\geq 100 \mathrm{mmHg}$, and 24 -h proteinuria $\geq 2 \mathrm{~g}$ ), with the average age of 31.6 years and median age of 30 years (ranging from 25 to 36 years). For the normal control group, the average age was 27.6 years and the median age was 26 years (ranging from 24 to 33 years). The pregnant females in both these two groups were singleton pregnancies.

These subjects reported no heart, liver, or kidney diseases; no blood system diseases; no diabetes, rheumatism, no autoimmune diseases; and no infection or medication history within 4 weeks before surgery. After the fetus was delivered, the placental tissue on the maternal side attached to the umbilical cord was harvested $(1 \times 1 \times 1 \mathrm{~cm})$. After rinsing with physiological saline, the tissue was ground in an ice bath to obtain a single-cell suspension. Prior written informed consent was obtained from every patient and the study was approved by the local ethics review board of the Laiwu Maternal and Child Health Hospital.

Culture of human trophoblast HTR8-SVneo cells. Human HTR8-SVneo trophoblast cells were purchased from the Cell Bank of the Chinese Academy of Sciences. The cells were cultured in RPMI-1640 medium (Thermo Fisher Scientific, Inc.) containing $10 \%$ fetal bovine serum (FBS). When $90 \%$ confluence was reached, the cells were passaged. HTR8-SVneo cells in the logarithmic growth phase were used for the following experiments.

Quantitative real-time PCR. Total RNA was extracted with Trizol (cat. no. B511311; Sangon Biotech Co., Ltd.). The cDNA template was obtained with RT-PCR. Quantitative real-time PCR was performed with the QuantiFast SYBR ${ }^{\circledR}$ Green PCR Kit (Qiagen). The 20- $\mu$ l PCR system consisted of $2 \mu \mathrm{l}$ cDNA, $10 \mu \mathrm{l}$ RT-qPCR-Mix, $0.5 \mu \mathrm{l}$ upstream primer (sequence: 5'-CAGCAGCAATTCATGTTTTGAA-3'), $0.5 \mu 1$ downstream primer (general primer provided within the kit),

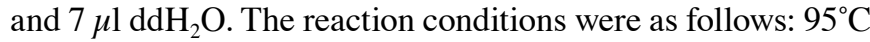
for $10 \mathrm{~min} ; 95^{\circ} \mathrm{C}$ for $1 \mathrm{~min}$, and $60^{\circ} \mathrm{C}$ for $30 \mathrm{sec}$, for a total of 40 cycles.

Cell transfection. For the cell transfection, $2 \times 10^{5}$ HTR8-SVneo cells in the logarithmic growth phase were seeded onto the 24-well plates, and cultured in RPMI-1640 medium containing $10 \%$ FBS without antibiotics. On the following day, transfection was performed for the cells with $70 \%$ confluence. The miR-338-3p mimics were synthesized by Hanbio (Shanghai, China). According to the manufacturer's instructions, $1.5 \mu \mathrm{l}$ $50 \mathrm{pmol} / \mu \mathrm{l} \mathrm{miR-338-3p} \mathrm{mimics} \mathrm{and} 1 \mu \mathrm{l}$ lipo2000 (Thermo Fisher Scientific, Inc.) was added into the EP tubes containing $50 \mu$ l Opti-MEM I medium (Thermo Fisher Scientific, Inc.), respectively, which was then mixed after $5 \mathrm{~min}$. After $20 \mathrm{~min}$ at room temperature, the mixture was used to incubate the cells for $6 \mathrm{~h}$. Then the cells were incubated with RPMI-1640 culture medium containing $10 \%$ FBS. For the viral transfection, the Lv-AKT3 viral vector was synthesized by Hanbio Co. The vector was used to transfect the cells at a multiplicity of infection (MOI) of 20 for $6 \mathrm{~h}$, and then the cells were cultured with the RPMI-1640 medium containing 10\% FBS. After $48 \mathrm{~h}$, the cells were harvested, and the biological functions and protein expression levels were detected.

CCK-8 assay. The proliferation of the HTR8-SVneo cells before and after transfection was assessed with the CCK-8 kit (Beyotime, Beijing, China), according to the manufacturer's instructions. In brief, HTR8-SVneo cells was seeded onto 96-well plates, at a density of $2 \times 10^{3}$ cells/well. After culture for $0,24,48$ and $72 \mathrm{~h}$, the cells were incubated with $20 \mu 15 \mathrm{~g} / \mathrm{l}$ CCK-8. On the last day, $150 \mu \mathrm{l}$ CCK-8 reaction solution was added to incubate the cells at $37^{\circ} \mathrm{C}$ for $2 \mathrm{~h}$, and the absorbance at $490 \mathrm{~nm}$ was measured. Cell proliferation curve was obtained accordingly.

Flow cytometry. After transfection, a total of $1 \times 10^{6}$ cells were harvested and washed with pre-cooled PBS. Cellular apoptosis was performed with the FITC Annexin V Apoptosis Detection kit I (BD Biosciences), according to the manufacturer's instruction. In the assay, the cells positive for Annexin $\mathrm{V}$ alone were considered as apoptotic cells, while the cells positive for PI alone were regarded as necrotic cells.

Transwell chamber assay. The ability of invasion and metastasis of the HTR8-SVneo cells was detected by Transwell chamber assay. Matrigel (BD Biosciences) was used to simulate the extracellular matrix environment. For the invasion assay, Matrigel was melted at $4^{\circ} \mathrm{C}$ overnight. On the next day, the Matrigel stock solution was diluted (1:2) with the serum-free RPMI-1640 medium. A total of $50 \mu \mathrm{l}$ of the mixture was evenly smeared onto the upper chamber, which was incubated at $37^{\circ} \mathrm{C}$ for $60 \mathrm{~min}$. A total of $1 \times 10^{5}$ cells were seeded onto the Transwell upper chamber containing $200 \mu \mathrm{l}$ serum-free RPMI-1640 medium, and $500 \mu$ l of the RPMI-1640 culture medium containing $10 \%$ FBS was added into the lower chamber. After $24 \mathrm{~h}$, the cells were fixed with $4 \%$ formaldehyde 
at room temperature for $10 \mathrm{~min}$. After washing with PBS, the cells in the upper chamber were wiped off, and then subjected to the Giemsa staining. The invading cells were observed under x200 magnification. For the migration assessment, the same procedures were performed, except that no Matrigel was added into the chamber. Under light microscopy, five fields were randomly selected, and the transmembrane cells were counted.

Laser confocal scanning microscopy (LCSM). Effects of miR-338-3p on the cytoskeleton were observed by LCSM. When the $50 \%$ confluence was reached, the culture medium was discarded. After washing, the cells were fixed with $4 \%$ paraformaldehyde at room temperature for $10 \mathrm{~min}$, following by permeabilization with $0.5 \%$ Triton X-100 at room temperature for $5 \mathrm{~min}$. After washing with PBS at room temperature for $30 \mathrm{sec}$, the cells were incubated with $200 \mu \mathrm{l} 100 \mathrm{nmol} / \mathrm{l}$ rhodamine in dark at room temperature for $30 \mathrm{~min}$. After counterstaining with $200 \mu 1100 \mathrm{nmol} / 1$ DAPI for $30 \mathrm{sec}$, the cytoskeleton was observed with LCSM.

Bioinformatics analysis. miR-338-3p was searched as the key word on the Targetscan www.targetscan.org website (Version 7.2), and its target genes were analyzed. The potential target genes for miR-338-3p were submitted to the online Database for Annotation, Visualization and Integrated Discovery (DAVID)(https://david.ncifcrf.Gov/; the species of human) (Version 5.1) to analyze the potential downstream signaling pathways.

Western blot analysis. Cells were collected and lysed with $1 \mathrm{ml}$ RIPA lysis on ice. Protein concentration was determined with the BCA method (Beyotime). Totally $10 \mu \mathrm{l}$ protein sample was separated with $10 \%$ SDS-PAGE, and then electronically transferred onto a PVDF membrane. After blocking with fat-free milk at room temperature for $1 \mathrm{~h}$, the membrane was incubated with rabbit anti-human anti-AKT primary antibody (1:1,000 dilution; AF1777; Beyotime) or mouse anti-human anti-GAPDH primary antibody (1:4,000 dilution; AF5009; Beyotime) at $4^{\circ} \mathrm{C}$ overnight. Then the membrane was incubated with HRP-conjugated goat anti-mouse or goat anti-rabbit secondary antibody (both at 1:4,000 dilution; A0208 and A0216, respectively; Beyotime) at room temperature for $1 \mathrm{~h}$. Color development was performed with the ECL method.

Dual luciferase reporter assay. Based on the bioinformatic prediction, the wild-type and mutant seed regions in the 3'-UTR (untranslated region) of the $A K T 3$ gene for miR-338-3p were synthesized, and the restriction sites for Spe 1 and HindIII were added to both ends, which was then cloned into the pMIR-REPORT luciferase reporter plasmid. Totally $0.5 \mu \mathrm{g}$ plasmid carrying the wild-type or mutant 3'-UTR DNA sequence was co-transfected with miR-338-3p mimics into $293 \mathrm{~T}$ cells with liposomes. After $24 \mathrm{~h}$, the cells were lysed and the luciferase activity was detected using the Double Luciferase Reporter AssaykKit (RG027; Beyotime Institute of Biotechnology), according to the kit instructions. Fluorescence was determined using the GloMax 20/20 luminometer (Promega Corporation). Renilla was used as internal reference.
Statistical analysis. Data are expressed as mean \pm SD (standard deviation). SPSS 17.0 software was used for statistical analysis. Experiments were performed independently three times. The unpaired t-test was performed for statistical analysis, and one-way ANOVA was conducted for the multi-group comparison, with the Turkey post hoc test. $\mathrm{P}<0.05$ was considered as indicative of statistical significance.

\section{Results}

Distribution of miR-338-3p in the peripheral blood and placenta of patients with PIH. In order to study the function and mechanism of miR-338-3p in patients with PIH, the expression levels of miR-338-3p in the peripheral blood and placenta of $\mathrm{PIH}$ were detected by quantitative real-time PCR. Our results showed that the mRNA expression levels of miR-338-3p were significantly elevated in the placental tissue of $\mathrm{PIH}(4.72 \pm 0.12$; $\mathrm{P}<0.05$ ) when compared to that in the normal tissues (Fig. 1A). The relative mRNA expression levels of miR-338-3p in the placental tissue of patients with severe pre-eclampsia, mild pre-eclampsia, and simple PIH were $6.15 \pm 0.27,4.30 \pm 0.11$, and $3.60 \pm 0.08$, respectively, with statistical significance between these groups $(\mathrm{P}<0.05$; Fig. 1B).

Furthermore, in the peripheral blood, the relative levels of miR-338-3p in patients with simple PIH, mild pre-eclampsia, and severe pre-eclampsia were $2.47 \pm 0.09,2.70 \pm 0.12$, and $3.80 \pm 0.14$, respectively. Statistical analysis showed that the miR-338-3p expression level was significantly elevated in the peripheral blood of the patients with severe pre-eclampsia, compared with the patients with simple PIH and mild pre-eclampsia $(\mathrm{P}<0.05$; Fig. $1 \mathrm{C})$. These results suggest that, along with the development of PIH, the expression levels of miR-338-3p in the placental tissue are significantly elevated, which might be associated with disease development.

miR-338-3p inhibits the proliferation of HTR8/SVneo cells in vitro. To investigate the biological functions of miR-338-3p, miR-338-3p mimics were transfected into the HTR8-SVneo cells. Our results from the quantitative real-time PCR showed that the mRNA expression level of miR-338-3p was significantly increased in the miR-338-3p mimic-transfected cells $(\mathrm{P}<0.05)$ when compared to the level in the miR-NC (negative control)-transfected cells (Fig. 2A). The results from the CCK-8 assay showed that, when the miR-338-3p expression level was elevated, the optical density $\mathrm{OD}_{490 \mathrm{~nm}}$ values of the HTR8/SVneo cells at 24, 48 and $72 \mathrm{~h}$ were significantly lower than the values of the miR-NC group $(\mathrm{P}<0.05)$, suggesting that enhanced expression of miR-338-3p suppressed cell proliferation activity (Fig. 2B).

Moreover, the results from flow cytometry showed that the number of HTR8/SVneo cells positive for the proliferation-related marker Ki-67 was significantly decreased after the miR-338-3p expression level was upregulated (Fig. 2C; $\mathrm{P}<0.05)$. These results indicate that miR-338-3p can inhibit the proliferation of HTR8/SVneo cells in vitro, and its high expression level in placental tissue may be one of the reasons for the inhibited HTR8/SVneo cell infiltration.

miR-338-3p inhibits the invasion and metastasis of HTR8/SVneo cells. The infiltration ability of trophoblast cells in 
A

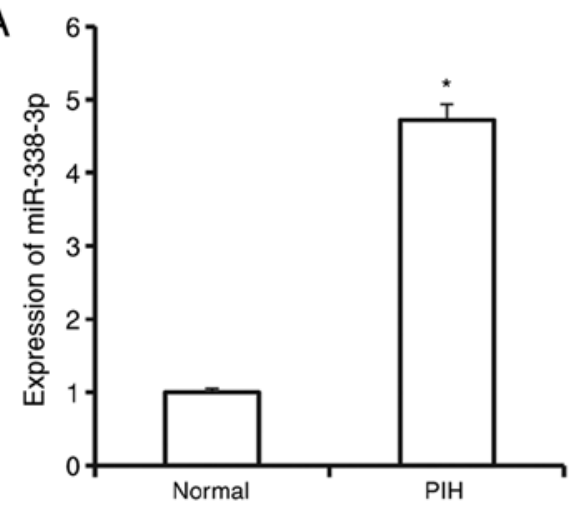

B

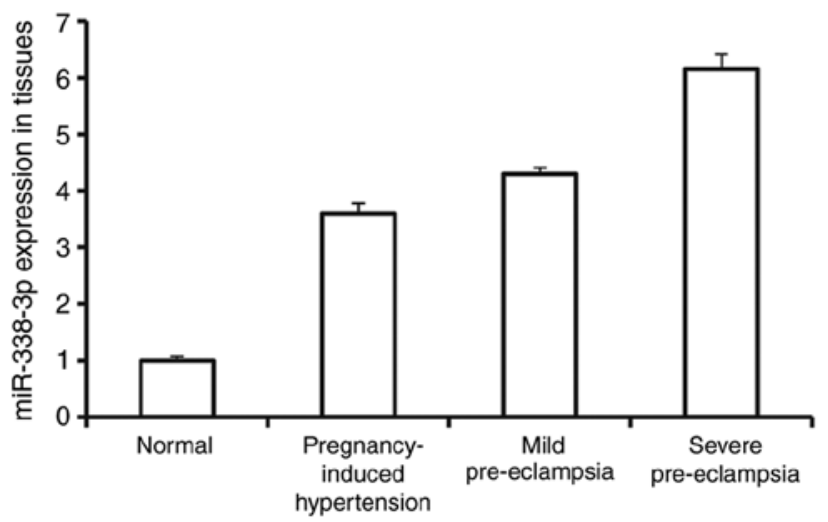

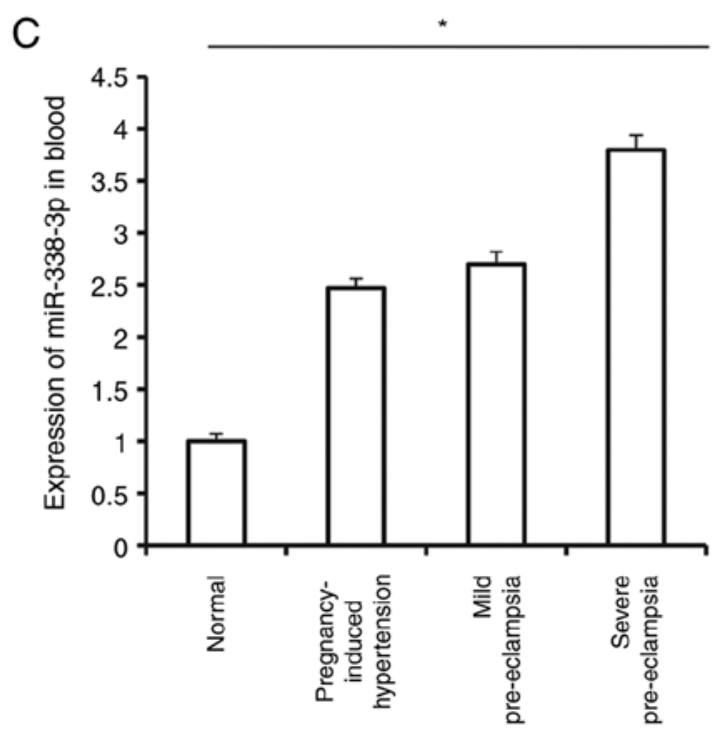

Figure 1. Expression of miR-338-3p in PIH. The expression levels of miR-338-3p in the peripheral blood and placental tissue were assessed by quantitative real-time PCR. (A) Elevated miR-338-3p expression levels in the placental tissue of PIH. (B) Positive relationship between miR-338-3p expression and the PIH development. (C) Elevated miR-338-3p levels in the peripheral blood of $\mathrm{PIH}$ patients. ${ }^{*} \mathrm{P}<0.05$. PIH, pregnancy-induced hypertension.

A

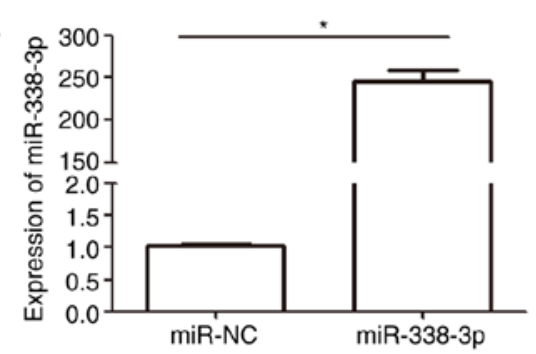

C

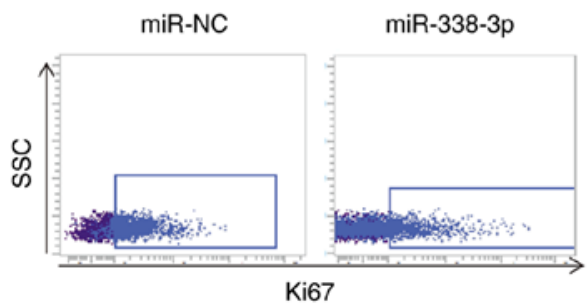

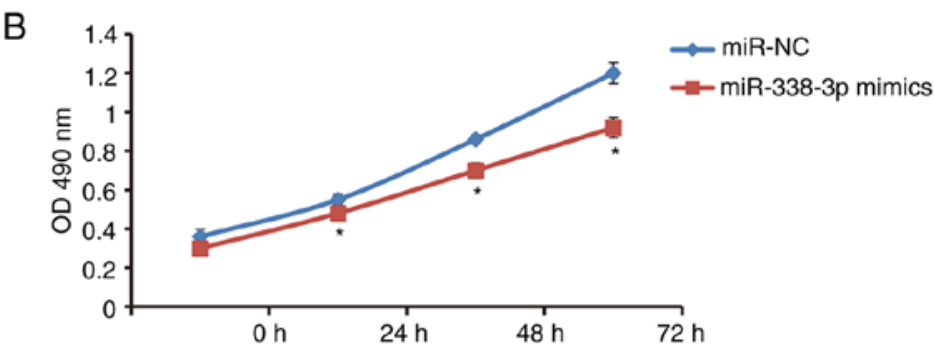

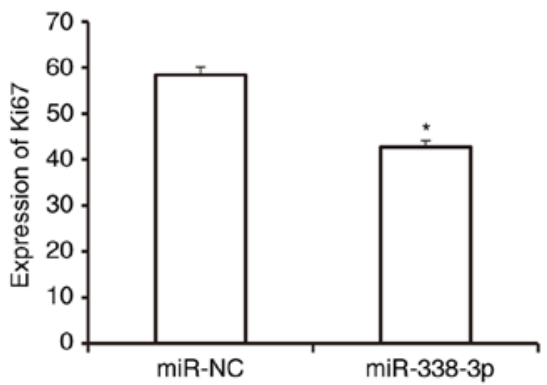

Figure 2. miR-338-3p inhibits the proliferation of trophoblast cells. (A) miR-338-3p expression was detected following transfection of the human HTR8-SVneo trophoblast cells with miR-338-3p mimics or miR-NC (negative control) by quantitative real-time PCR. (B) Effects of miR-338-3p on the proliferation of trophoblast cells were assessed with the CCK-8 assay. Comparison was performed between groups at each time point. (C) Ki67-positive cell rates were assessed with flow cytometry. ${ }^{*} \mathrm{P}<0.05$. 
A

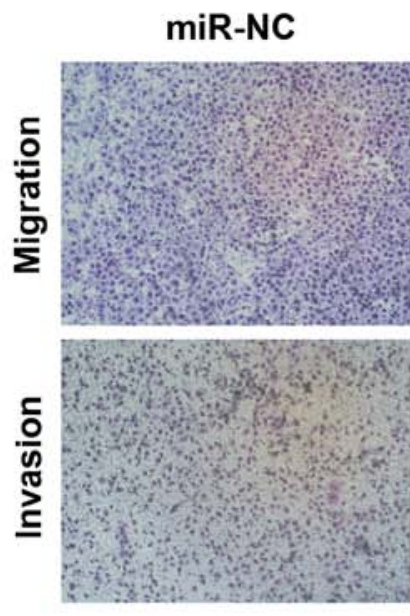

C

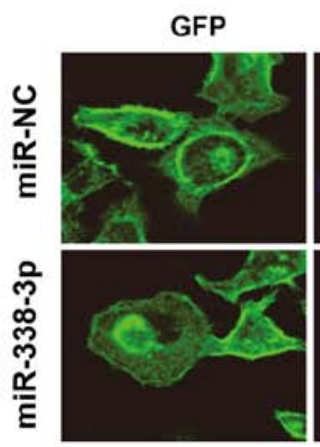

miR-338-3p

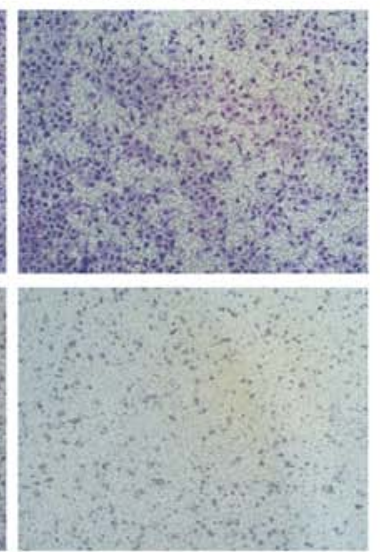

DAPI

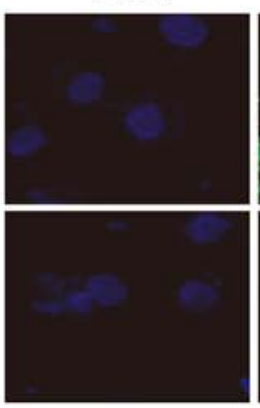

Merge

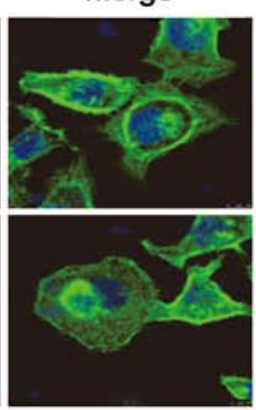

B

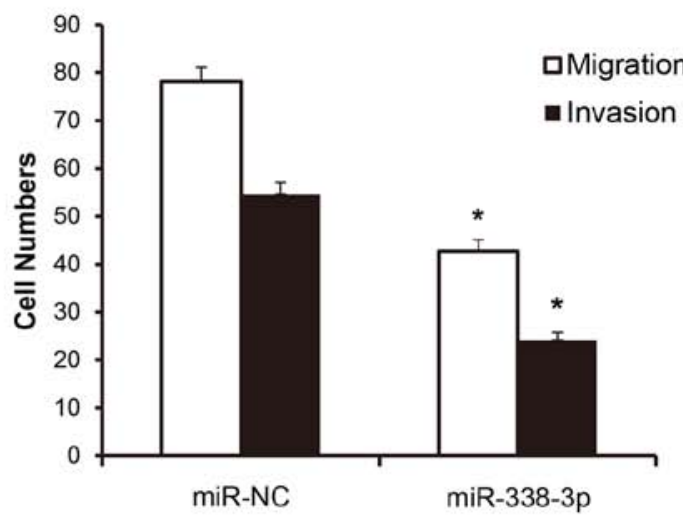

$\mathbf{D}$

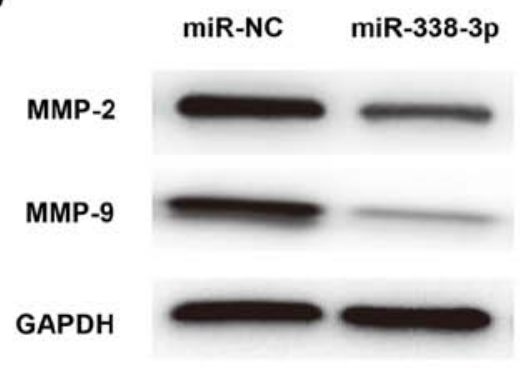

Figure 3. miR-338-3p inhibits the infiltration ability of trophoblast cells. (A and B) The invasion and migration abilities of human HTR8-SVneo trophoblast cells were assessed with Transwell chamber assays. (C) Trophoblast cell myofilaments were observed by LCSM. (D) The expression levels of MMP-2 and MMP-9 were detected by western blot analysis. "P<0.05 vs. the control group. LCSM, laser confocal scanning microscopy; MMP, matrix metalloproteinase.

the placental tissue is closely related to placental development. Therefore, the invasion and metastasis abilities of trophoblast cells were detected using the Transwell assay. Our results showed that accompanied with the upregulation of miR-338-3p expression, the migration ability of the HTR8/SVneo cells was significantly suppressed. The number of cells passing through the chamber membrane was significantly lower than the control group (42.7 \pm 2.4 vs. $78.2 \pm 2.9)$ (Fig. 3A and B). For assessment of the invasion ability, our results showed that, after the miR-338-3p expression level was elevated, the number of HTR8/SVneo cells crossing the Transwell chamber membrane was significantly decreased (26.1 \pm 1.6 vs. 54.6 \pm 2.4 ; Fig. 3A and B). LCSM results showed that, along with the upregulation of the expression of miR-338-3p, the myofilaments on the surface of the HTR8/SVneo cells were significantly reduced, and the fluorescence intensity was decreased, suggesting increased migratory capacity (Fig. 3C). Moreover, western blot analysis showed that the protein expression levels of matrix metalloproteinase-2 (MMP-2) and MMP-9 in the miR-338-3p mimic-transfected cells were significantly lower than levels in the miR-NC group, suggesting decreased invasion ability (Fig. 3D). These results revealed that upregulation of miR-338-3p inhibited the invasion and metastatic abilities of the HTR8/SVneo cells.

Effects of miR-338-3p on anoikis of HTR8/SVneo cells. Anoikis refers to the cell ability to detach from the in situ environment, and to exert anti-apoptosis activity during distant metastasis, which is also an important factor affecting the infiltration of HTR8/SVneo cells in the placental tissue. To simulate the anoikis environment, the HTR8/SVneo cells were transfected with the miR-338-3p mimics at $37^{\circ} \mathrm{C}$ for $24 \mathrm{~h}$. The cells were collected, and apoptosis was detected by flow cytometry. Our results showed that, after miR-338-3p mimic transfection, the apoptosis rate of the HTR8/SVneo cells was significantly increased compared with the miR-NC group (10.8 \pm 0.9 vs. $24.6 \pm 1.5$; P<0.05; Fig. 4). These results suggest that miR-338-3p promotes the apoptosis of HTR8/SVneo cells.

AKT3 is a target gene of $m i R-338-3 p$. Bioinformatic analysis showed that $A K T 3$ is a potential target gene of miR-338-3p (Fig. 5A), and the AKT3 downstream is involved in the multiple signaling pathways involved in the regulation of cell proliferation, apoptosis, and metastasis. Our results from the western blot analysis showed that the expression level of AKT3 in the miR-338-3p mimic-transfected group was decreased when compared with that in the miR-NC group (Fig. 5B) $(\mathrm{P}<0.05)$. Our results from the dual luciferase reporter assay showed that, compared with the control group, after co-transfection of the miR-338-3p mimics and pMIR-REPORT-wild-type luciferase reporter plasmids, the fluorescence in the cells was significantly downregulated $(\mathrm{P}<0.05)$, while no significant differences were observed in the fluorescence for the co-transfection of 

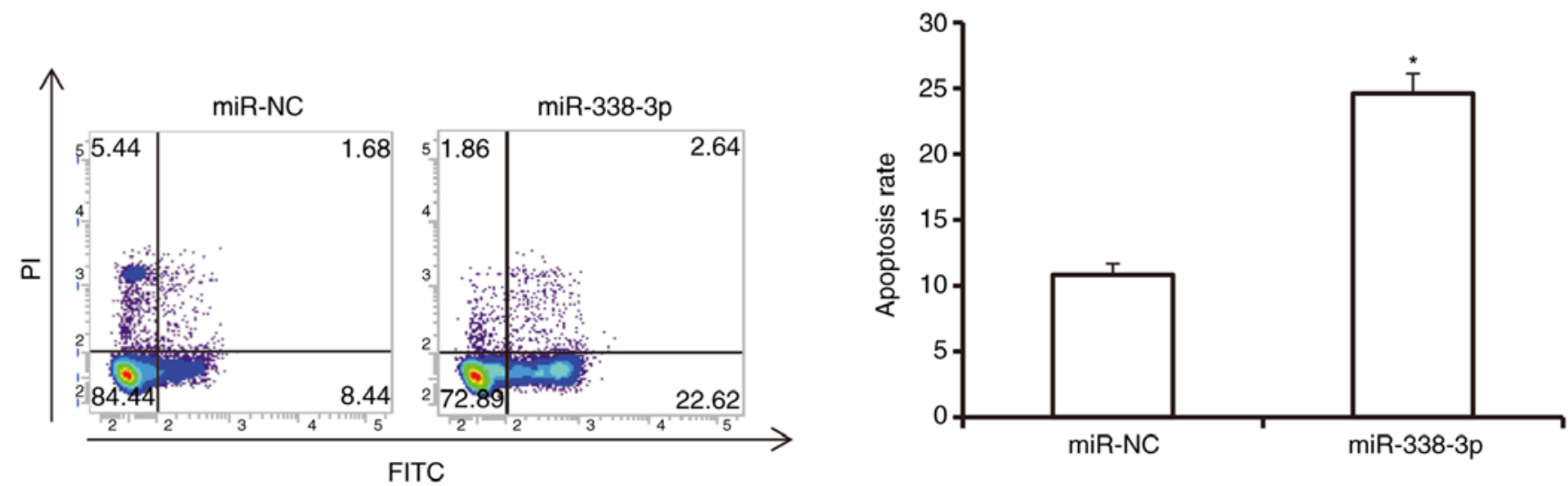

Figure 4. Effects of miR-338-3p on anoikis in the human HTR8-SVneo trophoblast cells. Effects of miR-338-3p on anoikis in trophoblast cells were detected by flow cytometry. ${ }^{*} \mathrm{P}<0.05$.

A Wild-type

$$
\begin{aligned}
& 5^{\prime} \quad \text {...CAAUCAGAUUGUGGCAUGCUGGG... } \\
& \text { I I | | | | | | | } \\
& \text { 3' GUUGUUUUAGUGACUACGACCU } \\
& \text { Mutant-type }
\end{aligned}
$$

5' ...CAAUCAGAUUGUGGCGUACUGAG...

3' GUUGUUUUAGUGACUACGACCU

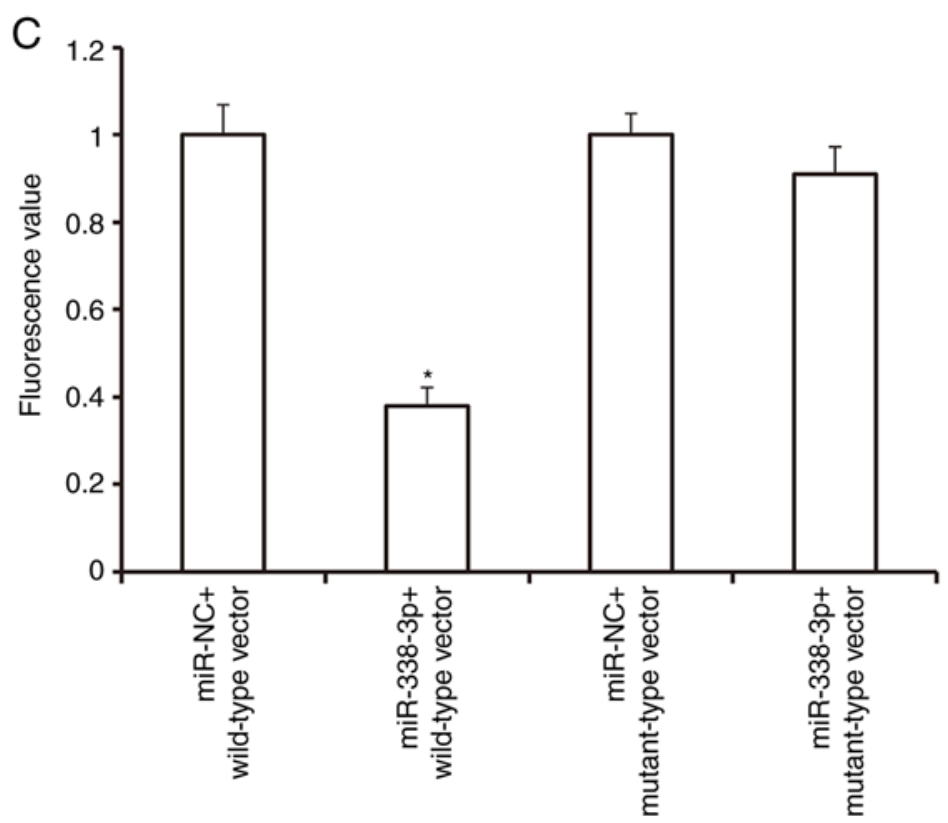

B

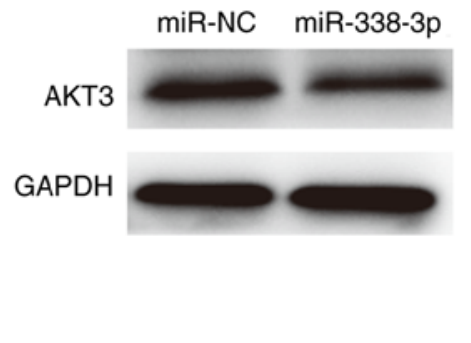

D miR-NC miR-338-3p Rescue LV-AKT3 - $\quad-\quad-\quad+$

AKT3

GAPDH

Figure 5. AKT3 is a target gene of miR-338-3p. (A) Bioinformatic analysis of the binding sites between miR-338-3p and AKT3. (B) miR-338-3p downregulated the expression of AKT3 in the human HTR8-SVneo trophoblast cells. (C) Dual luciferase reporter assay. NC, normal control group; and wild-type and mutant, groups carrying the wild-type and mutant 3 '-UTR DNA sequences, respectively. ${ }^{*} \mathrm{P}<0.05$ vs. the control group. (D) AKT3 expression was assessed by western blot analysis following transfection of Lv-ACT3. AKT, serine-threonine kinase [also known as protein kinase B (PKB)].

the pMIR-REPORT-mutant luciferase reporter plasmids and miR-338-3p mimics (Fig. 5C; P>0.05).

Moreover, whether AKT3 overexpression could restore the phenotype of HTR8/SVneo cells transfected with miR-338 mimics was further evaluated. Based on the transfection of miR-338 mimics, AKT3 was overexpressed. The AKT expression levels were detected and confirmed by western blot analysis (Fig. 5D). Our results of the rescue experiments following AKT overexpression showed significantly increased proliferation, decreased infiltration ability and decreased apoptosis of the HTR8/SVneo cells ( $\mathrm{P}<0.05$; Fig. 6), indicating that miR-338-3p exerts biological function by downregulating 

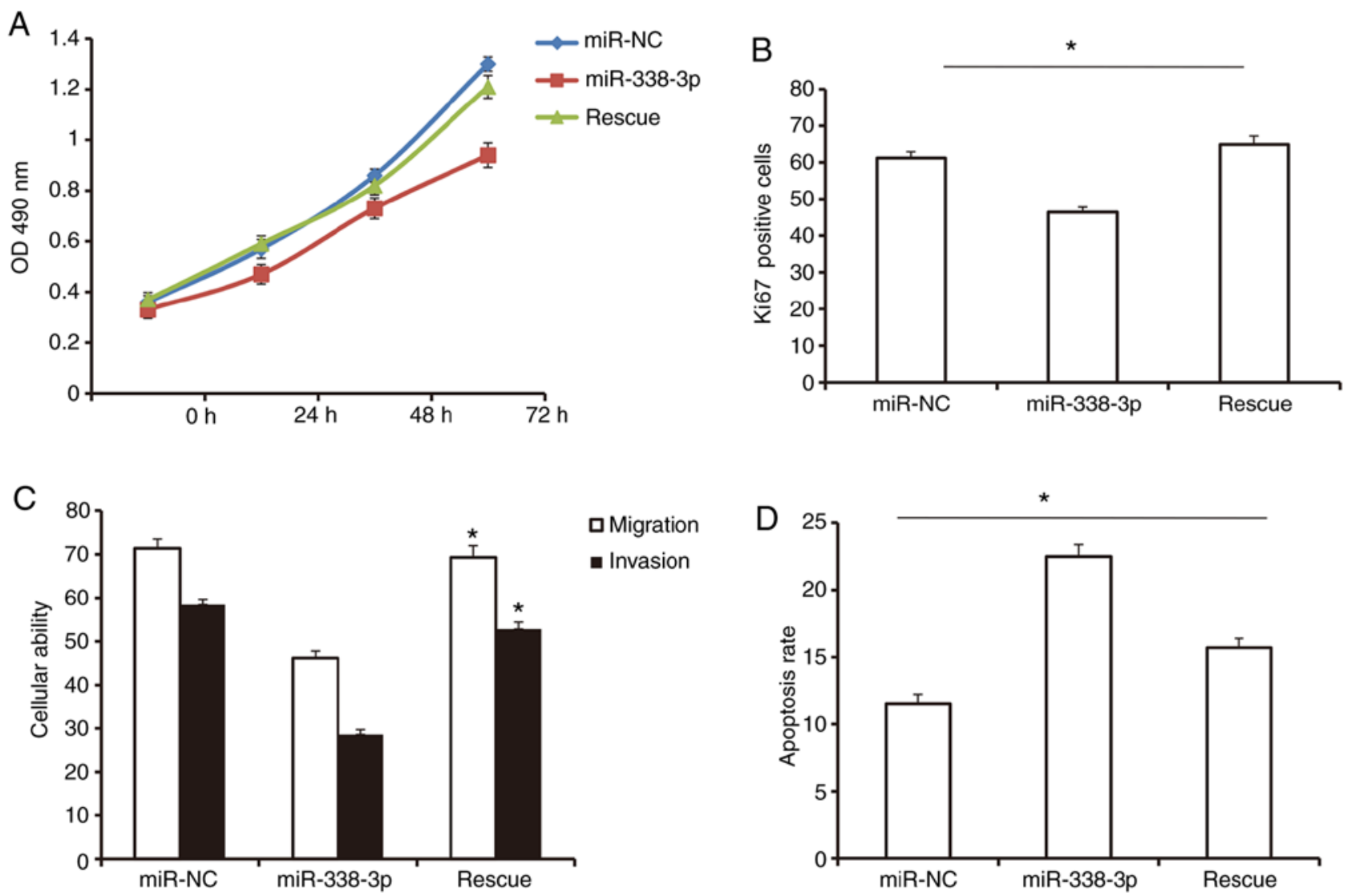

Figure 6. Effects of AKT3 on miR-338-3p-induced changes in human HTR8-SVneo trophoblast cells. (A and B) AKT3 upregulation reversed the inhibitory effects of miR-338-3p on trophoblast cell proliferation. There were 3 groups at each time point, and the ANOVA test was carried out. (C) AKT3 upregulation reversed the inhibitory effects of miR-338-3p on invasion and metastasis of trophoblast cells. (D) AKT3 upregulation restored the inhibiting effects of miR-338-3p on anoikis in trophoblast cells. ${ }^{*} \mathrm{P}<0.05$. AKT, serine-threonine kinase [also known as protein kinase B (PKB)].

the AKT3 expression levels in the HTR8/SVneo cells. These results suggest that miR-338-3p is able to bind to the $3^{\prime}$-UTR of AKT3 mRNA, i.e., its target gene.

\section{Discussion}

Hypertensive disorders during pregnancy are common obstetric complications in the clinic, which are unique to pregnant women during pregnancy (19). After 20 weeks of gestation, patients are characterized by clinical features such as obvious hypertension and different degrees of proteinuria. If not timely treated, this hypertension may be a great threat to the lives of the mother and fetus, even death (8). At present, the pathogenesis of pregnancy-induced hypertension (PIH) remains unclear. Current research shows that PIH is mostly transient. After the placenta is delivered, the symptoms of hypertension in most patients disappear, and there is no obvious sequelae, suggesting that the placenta may participate in the regulation of PIH (20). Trophoblast cells play important roles in the formation and function of the placenta, and its biological abnormalities are associated with abnormal placental functions. The mechanisms by which miRNAs regulate the biological functions of trophoblast cells remain unclear. In the present study, our results revealed that miR-338-3p was significantly elevated in the placental tissue and peripheral blood of PIH patients, which is positively correlated with disease development. The in vitro experiments showed that overexpression of miR-338-3p inhibiting the proliferation and the invasion/metastasis abilities of human trophoblast HTR8/SVneo cells by downregulated AKT3 expression.

It has been previously shown that placental growth and development are directly related to the success or failure of pregnancy, in which the normal development of blood vessels in the placenta is the key to ensure that the fetus receives sufficient oxygen and nutrients from the mother (21). However, the regulatory mechanism of these processes is extremely complex. Trophoblasts are the main cells that make up the placenta and are involved in the regulation of placental vascular remodeling. During normal pregnancy, trophoblasts undergo rapid proliferation and simultaneously invade the decidua to form a column of extracellular trophoblast cells, which in turn invades into the deep layers of the mesenchyme (to the interstitium at the upper one-third site of the myometrium) and the spiral arterioles. This induces the physiological revascularization of the spiral arterioles for the first time, thus causing the formation of the low-resistance, high-flow sinus vessels. During the first week of pregnancy, the proliferation and infiltration of intravascular trophoblast cells exhibit a second peak, infiltrating into the uterine muscle segment of the spiral arteriole, while the trophoblasts infiltrate the vessel wall to expand the spiral arteriole, completing the second revascularization process $(22,23)$. Therefore, trophoblast cells with normal biological functions are critical for placental remodeling. It has been reported that the abnormal 
expression of miRNAs in the placental tissues could affect the remodeling of the placenta. miR-4421 has been shown to regulate the progression of pre-eclampsia by targeting CYP11B2 (24). Moreover, miR-134 was found to inhibit the infiltration of placental trophoblast cells by targeting ITGB1, thus participating in the development of PIH (25). In recent years, miR-338-3p has been recognized as a tumor-associated miRNA, which suppresses the tumor cell function in invasion and metastasis processes (26). But whether miR-338-3p regulates the proliferation and infiltration of trophoblast cells has not yet been reported. In the present study, our results showed that miR-338-3p is upregulated in the placental tissue and peripheral blood of PIH patients and is positively correlated with the developmental of PIH. Further in vitro experiments revealed that upregulation of miR-338-3p expression inhibited the proliferation, invasion/metastasis, and apoptosis resistance of HTR8/SVneo trophoblast cells.

AKT (serine-threonine kinase), also known as protein kinase B (PKB), consists of approximately 480 amino acids (27). Studies have confirmed that AKT plays key roles in multiple signaling pathways, such as PI $3 \mathrm{~K}$ and $\mathrm{NF}-\kappa \mathrm{B}$, and inhibits apoptosis and promotes cell proliferation by regulating the transcription of various downstream factors $(28,29)$. Three subtypes of AKT are currently found in mammals, i.e., AKT1, AKT2, and AKT3, encoded by three different genes located on chromosomes 14q32, 19q13, and 1q43, respectively, which all contain a kinase catalytic domain, an amino-terminal $\mathrm{PH}$ domain, and a carboxy terminal regulatory domain (30). AKT3 can promote cell proliferation and metastasis, and inhibit cellular apoptosis. It has been shown that AKT3 promotes the survival of embryonic stem cells (31). Moreover, AKT3 was found to promote the development of adenoid cystic carcinoma (32). In addition, miRNAs play important roles in the regulation of AKT3. It has been found that miR-145 targets AKT3 to inhibit inflammatory responses and relieve neuralgia (33). Moreover, miR-433 was found to inhibit breast cancer proliferation and metastasis by targeting AKT3 (34). These results indicate that miRNAs are involved in the regulation of AKT3 expression. In the present study, our results from the bioinformatic analysis showed that AKT3 is a potential target gene of miR-338-3p. Moreover, our results from the western blot analysis and dual luciferase reporter assay showed that miR-338-3p inhibits the expression level of AKT3. In addition, upregulation of AKT3 expression restored the miR-338-3p-induced inhibitory effects on the biological functions of trophoblast cells, suggesting that miR-338-3p regulates the trophoblast cell proliferation and metastasis/apoptosis, via AKT3. Although AKT3 is involved in the regulation of various downstream pathways, our results confirmed that miR-338-3p regulates the biological functions of trophoblast cells through AKT3. However, further in-depth studies are still required to elucidate the major downstream regulatory pathways involved herein.

In conclusion, our results showed that the upregulated miR-338-3p expression demonstrated in PIH is associated with disease development. Moreover, miR-338-3p suppressed the proliferation and infiltration, and increases the apoptosis of trophoblast cells by regulating the expression level of AKT3, thus affecting the development of the placenta. These findings provide a potential clinical therapeutic target for PIH.

\section{Acknowledgements}

We thank Director Aixia Zou (Laiwu Maternal and Child Health Hospital, Laiwu, Shandong, China) and Dr Jichao Liu (The First Affiliated Hospital of Zhengzhou University, Zhengzhou, Henan, China) for their assistance in the study design, data collection and analysis, and manuscript preparation.

\section{Funding}

No funding was received.

\section{Availability of data and materials}

The datasets used and/or analyzed during the present study are available from the corresponding author on reasonable request.

\section{Authors' contributions}

JL, YW and HL designed the current study, performed the experiments, collected and analyzed the data, and prepared the manuscript. All authors read and approved the final manuscript.

\section{Ethics approval and consent to participate}

Prior written informed consent was obtained from every patient and the study was approved by the local Ethics Review Board of the Laiwu Maternal and Child Health Hospital.

\section{Patient consent for publication}

Not applicable.

\section{Competing interests}

The authors declare that they have no competing interests.

\section{References}

1. Arendt LH, Henriksen TB, Lindhard MS, Parner ET, Olsen J and Ramlau-Hansen CH: Hypertensive disorders of pregnancy and genital anomalies in boys: A Danish Nationwide Cohort Study. Epidemiology 29: 739-748, 2018.

2. Brown MA, Magee LA, Kenny LC, Karumanchi SA, McCarthy FP, Saito S, Hall DR, Warren CE, Adoyi G and Ishaku S: International society for the study of hypertension in pregnancy (ISSHP): Hypertensive disorders of pregnancy: ISSHP classification, diagnosis, and management recommendations for international practice. Hypertension 72: 24-43, 2018.

3. West J, Lawlor DA, Santorelli G, Collings P, Whincup PH, Sattar NA, Farrar D and Wright J: Associations of social and economic and pregnancy exposures with blood pressure in UK White British and Pakistani children age 4/5. Sci Rep 8: 8966, 2018.

4. Li L, Zhao K, Hua J and Li S: Association between sleep-disordered breathing during pregnancy and maternal and fetal outcomes: An updated systematic review and meta-analysis. Front Neurol 9: 91, 2018.

5. Hill W, Holy R and Traiger G: EXPRESS: Intimacy, contraception, and pregnancy prevention in patients with pulmonary arterial hypertension: Are we counseling our patients? Pulm Circ: Jun 12, 2018 (Epub ahead of print).

6. Calimag-Loyola APP and Lerma EV: Renal complications during pregnancy: In the hypertension spectrum. Dis Mon 65: 25-44, 2019. 
7. Hu J, Wang H, Hu YF, Xu XF, Chen YH, Xia MZ, Zhang C and $\mathrm{Xu}$ DX: Cadmium induces inflammatory cytokines through activating Akt signaling in mouse placenta and human trophoblast cells. Placenta 65: 7-14, 2018

8. Wang H, Wang T, Dai L, Cao W, Ye L, Gao L, Zhou B and Zhou R: Effects of CXCL3 on migration, invasion, proliferation and tube formation of trophoblast cells. Placenta 66: 47-56, 2018

9. Zhao HJ, Chang HM, Zhu H, Klausen C, Li Y and Leung PCK Bone morphogenetic protein 2 promotes human trophoblast cell invasion by inducing activin A production. Endocrinology 159 2815-2825, 2018.

10. Huang DJ, Huang ZM, Xiao HY, Wu ZK, Tang LJ and Jiang JH: Protein scaffolded DNA tetrads enable efficient delivery and ultrasensitive imaging of miRNA through crosslinking hybridization chain reaction. Chem Sci 9: 4892-4897, 2018.

11. Tang J, Zhang Z, Jin X and Shi H: miR-383 negatively regulates osteoblastic differentiation of bone marrow mesenchymal stem cells in rats by targeting Satb2. Bone 114: 137-143, 2018.

12. Biró O, Alasztics B, Molvarec A, Joó J, Nagy B and Rigó J Jr: Various levels of circulating exosomal total-miRNA and miR-210 hypoxamiR in different forms of pregnancy hypertension. Pregnancy Hypertens 10: 207-212, 2017.

13. Mei Z, Huang B, Mo Y and Fan J: An exploratory study into the role of miR-204-5p in pregnancy-induced hypertension. Exp Ther Med 13: 1711-1718, 2017.

14. Wang N, Feng Y, Xu J, Zou J, Chen M, He Y, Liu H, Xue M and Gu Y: miR-362-3p regulates cell proliferation, migration and invasion of trophoblastic cells under hypoxia through targeting Pax3. Biomed Pharmacother 99: 462-468, 2018

15. Sun F, Yu M, Yu J, Liu Z, Zhou X, Liu Y, Ge X, Gao H, Li M, Jiang $\mathrm{X}$, et al: miR-338-3p functions as a tumor suppressor in gastric cancer by targeting PTP1B. Cell Death Dis 9: 522, 2018.

16. Hua FF, Liu SS, Zhu LH, Wang YH, Liang X, Ma N and Shi HR: MiRNA-338-3p regulates cervical cancer cells proliferation by targeting MACC1 through MAPK signaling pathway. Eur Rev Med Pharmacol Sci 21: 5342-5352, 2017.

17. Xiao G, Wang Q, Li B, Wu X, Liao H, Ren Y and Ai N MicroRNA-338-3p suppresses proliferation of human liver cancer cells by targeting SphK2. Oncol Res 26: 1183-1189, 2018

18. Che G, Wang Y, Zhou B, Gao L, Wang T, Yuan F and Zhang L: Knockdown of heparanase suppresses invasion of human trophoblasts by activating p38 MAPK signaling pathway. Dis Markers 2018: 7413027, 2018

19. Li X, Wu C, Shen Y, Wang K, Tang L, Zhou M, Yang M, Pan T, Liu X and Xu W: Ten-eleven translocation 2 demethylates the $M M P 9$ promoter, and its down-regulation in preeclampsia impairs trophoblast migration and invasion. J Biol Chem 293 10059-10070, 2018.

20. Monteiro LJ, Cubillos S, Sanchez M, Acuña-Gallardo S, Venegas P, Herrera V Lam EW, Varas-Godoy M and Illanes SE: Reduced FOXM1 expression limits trophoblast migration and angiogenesis and is associated with preeclampsia. Reprod Sci 26: 580-590, 2019

21. Tateishi A, Ohira S, Yamamoto Y and Kanno H: Histopathological findings of pregnancy-induced hypertension: Histopathology of early-onset type reflects two-stage disorder theory. Virchows Arch 472: 635-642, 2018
22. Burton GJ and Jauniaux E: Development of the human placenta and fetal heart: Synergic or independent? Front Physiol 9: 373, 2018.

23. Velicky P, Windsperger K, Petroczi K, Pils S, Reiter B, Weiss T, Vondra S, Ristl R, Dekan S, Fiala C, et al: Pregnancy-associated diamine oxidase originates from extravillous trophoblasts and is decreased in early-onset preeclampsia. Sci Rep 8: 6342, 2018.

24. Gao X, Li H and Wei JX: MiR-4421 regulates the progression of preeclampsia by regulating CYP11B2. Eur Rev Med Pharmacol Sci 22: 1533-1540, 2018.

25. Qin Q, Wei F, Zhang J and Li B: miR-134 suppresses the migration and invasion of non-small cell lung cancer by targeting ITGB1. Oncol Rep 37: 823-830, 2017.

26. Zhang X, Wang C, Li H, Niu X, Liu X, Pei D, Guo X, Xu X and Li Y: miR-338-3p inhibits the invasion of renal cell carcinoma by downregulation of ALK5. Oncotarget 8: 64106-64113, 2017.

27. Wu Y, Wang M, Zhang K, Li Y, Xu M, Tang S, Qu X and Li C: Lactate enhanced the effect of parathyroid hormone on osteoblast differentiation via GPR81-PKC-Akt signaling. Biochem Biophys Res Commun 503: 737-743, 2018.

28. Li Y, Gong Q, Guo W, Kan X, Xu D, Ma H, Fu S and Liu J: Farrerol relieve lipopolysaccharide (LPS)-induced mastitis by inhibiting AKT/NF- $\mathrm{BB}$ p65, ERK1/2 and P38 signaling pathway. Int J Mol Sci 19: pii: E1770, 2018.

29. Justin-Thenmozhi A, Dhivya Bharathi M, Kiruthika R, Manivasagam T, Borah A and Essa MM: Attenuation of aluminum chloride-induced neuroinflammation and caspase activation through the AKT/GSK-3 $\beta$ pathway by hesperidin in Wistar rats. Neurotox Res 34: 463-476, 2018.

30. Wu X, Gowda NM, Kawasawa YI and Gowda DC: A malaria protein factor induces IL-4 production by dendritic cells via PI3K-Akt-NF- $\kappa$ B signaling independent of MyD88/TRIF and promotes Th2 response. J Biol Chem 293: 10425-10434, 2018.

31. Wang L, Huang D, Jiang Z, Luo Y, Norris C, Zhang M, Tian X and Tang Y: Akt3 is responsible for the survival and proliferation of embryonic stem cells. Biol Open 6: 850-861, 2017.

32. Zboray K, Mohrherr J, Stiedl P, Pranz K, Wandruszka L, Grabner B, Eferl R, Moriggl R, Stoiber D, Sakamoto K, et al: AKT3 drives adenoid cystic carcinoma development in salivary glands. Cancer Med 7: 445-453, 2018

33. Shi J, Jiang K and Li Z: MiR-145 ameliorates neuropathic pain via inhibiting inflammatory responses and mTOR signaling pathway by targeting Akt3 in a rat model. Neurosci Res 134: $10-17,2018$

34. Hu X, Wang J, He W, Zhao P and Ye C: MicroRNA-433 targets AKT3 and inhibits cell proliferation and viability in breast cancer. Oncol Lett 15: 3998-4004, 2018. International (CC BY-NC-ND 4.0) License. 